\title{
Probing thermotropic phase behavior of DPPC bilayers from electrical and topographic data in a horizontal black lipid membrane model (hBLM).
}

\author{
Natalia A. Corvalán ${ }^{1,2^{*}}$ and María A. Perillo ${ }^{1,2^{*}}$ \\ ${ }^{1}$ Universidad Nacional de Córdoba, Facultad de Ciencias Exactas, Físicas y Naturales. Departamento \\ de Química, Cátedra de Química Biológica. Córdoba, Argentina. \\ ${ }^{2}$ CONICET, Instituto de Investigaciones Biológicas y Tecnológicas (IIByT). Córdoba, Argentina \\ *Corresponding author: \\ natalia.corvalan@gmail.com \\ mperillo@unc.edu.ar
}

CONTENTS (S1 to S3 refer to page numbers)

S1 Title, authors, affiliations and description of supporting information content.

S2 Surface pressure-mean molecular area isotherms of DPPC and DPPC $/ n$-decane monomolecular layers at the air water interface.

S4 Arrhenius plots

S5 Analysis of membrane capacitance

S6 Current fluctuation patterns, self-similarity parameter $(\alpha)$ and histograms of the amplitude of the electrical current across DPPC-hBLM under the percolation voltage intensity $\left(\mathrm{V}_{\text {th }}\right)$.

S7 Detrended fluctuation analysis of temporal I patterns.

S7. References. 


\section{Effect of the $\boldsymbol{n}$-decane content in DPPC monolayers on the surface pressure - mean molecular area ( $\pi$-Mma) isotherms.}

\section{Surface Pressure-Area Isotherms of DPPC}

Surface pressure-mean molecular area compression isotherms were obtained for pure or mixed lipid monolayers as described before (Suppl. Information in Corvalán et al., BBA Biomembranes 2013). Typically, monolayers were prepared by spreading lipids from $1 \mu \mathrm{mol} / \mathrm{ml}$ solutions in chloroform/methanol (2:1 v/v) over an unbuffered aqueous surface, contained in a teflon trough with an initial area of $242.25 \mathrm{~cm}^{2}$.

Before each experiment the trough was rinsed and wiped with $70 \% \mathrm{v} / \mathrm{v}$ ethanol and several times with bi-distilled water. The absence of surface-active compounds in pure solvents and in the subphase solution (bi-distilled water) was checked before each run by reducing the available surface area to less than $10 \%$ of its original value after enough time was allowed for the adsorption of possible impurities that might have been present in trace amounts. All measurements were performed at room temperature $(23 \pm 1 \circ \mathrm{C})$.

The film was relaxed about $5 \mathrm{~min}$ at $0 \mathrm{mN} / \mathrm{m}$ to allow the evaporation of the solvent and subsequently compressed to the target pressure. Surface pressure and film area were continuously measured and recorded with a computer controlled commercial device (Minitrough II, KSV Instruments Ltd., Finland) enclosed in a Plexiglas box to reduce surface contamination. The monolayers were compressed with two barriers that were moved synchronously by electronic switching. at a constant low rate of $5 \mathrm{mN} \cdot \mathrm{m}^{-1} \cdot \mathrm{min}^{-1}$. A lower compression rate $\left(1 \mathrm{mN} \cdot \mathrm{m}^{-1} \cdot \mathrm{min}^{-1}\right)$ was tested with identical results. The signal corresponding to the surface area (automatically

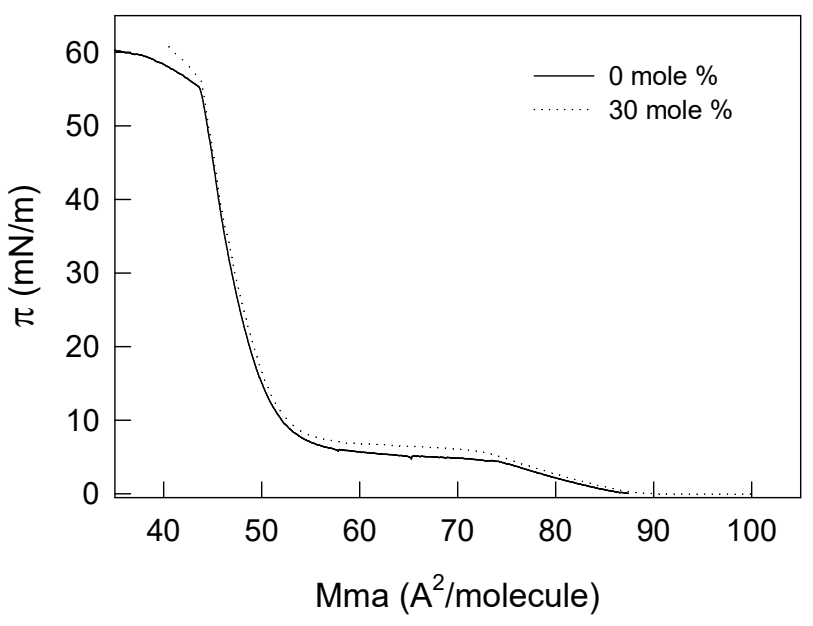

Fig.S1 Surface pressure $(\pi)$ vs. mean molecular area (Mma) compression isotherms. Full line, DPPC; dotted line, binary mixture of DPPC containing 30 mole determined by the Minitrough according to $\% n$-decane.

the relative position of the two compression barriers) and the output from the surface pressure transducer (measured automatically by the Minitrough with a Wilhelmy plate, platinized Pt foil $5 \mathrm{~mm}$ wide x 20 $\mathrm{mm}$ long $\mathrm{x} 0.025 \mathrm{~mm}$ thick) were fed into a personal computer through a serial interface using a specific software. 
Since $n$-decane lacks surface activity, its Mma was taken as $0 \AA^{2} /$ molec. Thus, mean molecular area (Mma) at varying pressures was calculated dividing surface area by the number of DPPC molecules spread on the interface.

Fig.S1 shows the pure DPPC $\pi$-Mma compression isotherm with the LE-LC bi-dimensional phase transition at $\pi \sim 5 \mathrm{mN} / \mathrm{m}$ described previously. The isotherm of DPPC containing 30 mole $\%$ of $n$-decane was superimposed with the former indicating that $n$-decane was mostly excluded from the monolayer phase. 


\section{Arrhenius plots}

The dependence of the Current Amplitude (I) and the membrane Resistance (R) on the temperature is shown by the Arrhenius plots in Fig. S2. Main and pretransition temperatures of the DPPC are evidenced as discontinuities in the $\ln (I)$ vs $T^{-1}$ (a) and $\ln (R) v s . T^{-1}$ curves, where the temperature dependence of $\mathrm{I}$ (and $\mathrm{R}$ ) is given by the $-E a / R$ factor corresponding to the slope of the natural logarithm transformation of Arrhenius equation:

$$
\ln I=\ln A-\frac{E_{a}}{R}\left(\frac{1}{T}\right)
$$

This equation is applied for determining the energy of activation of vast variety of thermallyinduced processes and reactions. In our experimental system, $E_{a}$ could be interpreted as the energy required for the current conducting events which involves a sequence of events that have been evidenced by molecular dynamics simulations (Tieleman 2004, Tarek 2005), and could be summarized as follows: 1) water molecule wires penetrating the hydrophobic core of the bilayer, apparently favored by local defects in the lipid headgroup region; then 2) the water wires growth and pores stabilization by lipid headgroups that migrate from the membrane-water interface to the middle of the bilayer. So, lower activation energy, which in our experimental model can be interpreted as the energy required for membrane permeation, is required at temperatures above the main transition temperature, while a higher permeation energy is required in the ordered phases. In turn, the lower permeation energy observed in
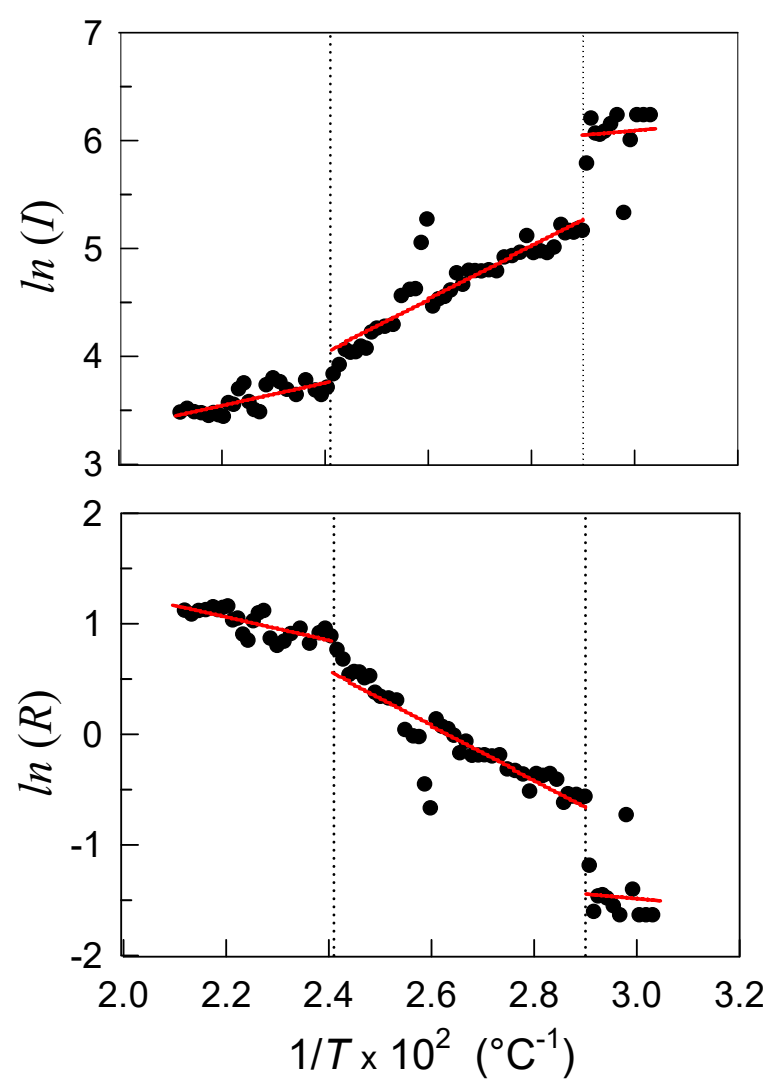

the gel phase with respect to the ripple phase could be attributed to the exacerbated susceptibility of the bilayer in gel phase $\left(\mathrm{L}_{\beta}\right)$ to the external electrical perturbation due to an electrostrictive effect of voltage.

Fig.S2. Arrhenius plots. Discontinuities in the $\ln (I)$ vs $1 / T$ (a) and $\ln (R)$ vs. $1 / T$ (b) plots correspond to first order transitions. Dotted lines indicate the main $\left(1 / \mathrm{T}_{\mathrm{P} \beta} \rightarrow \mathrm{L} \alpha=\right.$ $\left.0.0241^{\circ} \mathrm{C}^{-1} \equiv 41.49^{\circ} \mathrm{C}\right)$ and pretransition $\left(1 / \mathrm{T}_{\mathrm{L} \beta \rightarrow \mathrm{P} \beta}=0.029^{\circ} \mathrm{C}^{-1} \equiv 34.5^{\circ} \mathrm{C}\right)$ temperatures. 
Analysis of membrane capacitance

Capacitance (C) may provide information about membrane structure and geometry, commonly used as a control measurement for the correct bilayer formation. In the present work a minimum of 40 $\mathrm{pF}$ (in DPPC-BLMh at $0 \mathrm{mV}$ ) and maximum $115 \mathrm{pF}$ (in DPPC-hBLM at $160 \mathrm{mV}$ ) were found (Fig. $\mathrm{S} 2 \mathrm{a}$ ), equivalent to 0.23 and $0.65 \mu \mathrm{F} / \mathrm{cm}^{2}$ specific capacitances, respectively. These values are in accordance to those reported by Antonov et al. (Antonov, Anosov et al. 2003) who found an average specific capacitance of the hydrogenated egg-lecithin BLM of $0.73 \mu \mathrm{F} / \mathrm{cm}^{2}$, which was nearly twice as much as that for BLM formed from native egg lecithin. Voltage induced changes in membrane capacitance is consistent with what was previously reported by our group and others (Toyama, Nakamura et al. 1991, Corvalán, Kembro et al. 2013) and corresponds to an increase in the polarity of the membrane core.

A decrease in the DPPC-hBLM capacitance by 15 to $30 \%$ was measured during cooling between $44^{\circ}$ to $34^{\circ} \mathrm{C}$ (Fig.S2b). From the mathematical expression that defines the membrane capacitance: $\mathrm{C}=$ $\varepsilon_{0} \cdot \varepsilon \cdot \mathrm{A} / \mathrm{d}$, where $\varepsilon_{0}$ is the permittivity in vacuum, $\varepsilon$ the permittivity, $\mathrm{d}$ the bilayer hydrocarbon thickness and $\mathrm{A}$ the area, it can be concluded that the change in capacitance $\mathrm{C}$ with temperature indicates an increase in the thickness of the hBLM. This result is consistent with those previously reported in

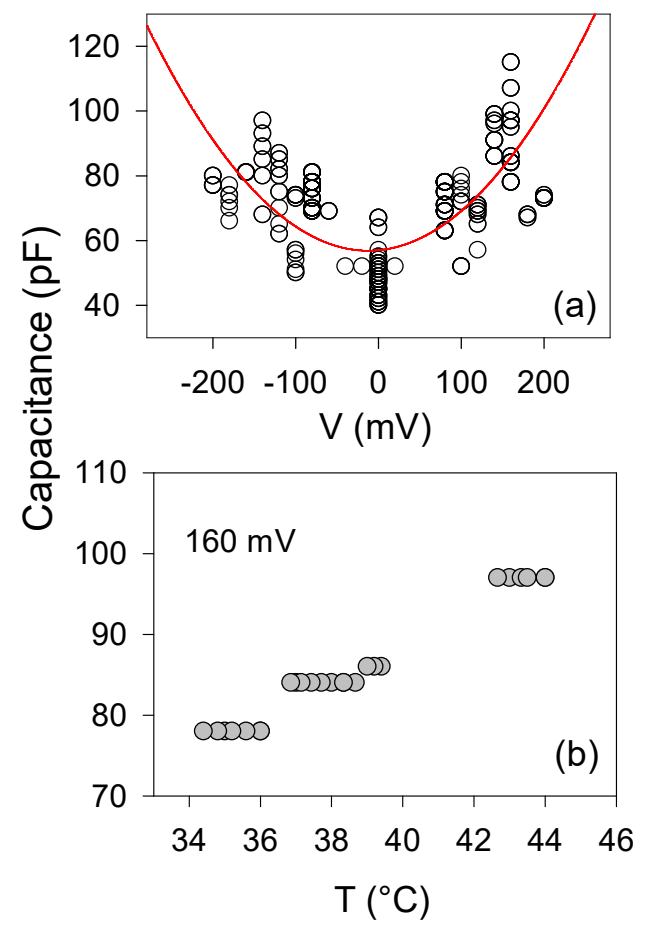

Fig.S3 Change in membrane capacitance $(\boldsymbol{C m})$. Effect of $\Delta V$ from 0 to $200 \mathrm{mV}$ in DPPC$\mathrm{hBLM}$ at temperatures between 32 and $48^{\circ} \mathrm{C}$ (a). Effect of temperature on $C$ recorded at $160 \mathrm{mV}$ from two independent bilayers (b). hydrogenated egg-lecithin BLM (Antonov, Anosov et al. 2003) and in X-ray diffraction from dispersions of bimolecular layers of dipalmitoyl phosphatidylcholine (Wilkins, Blaurock et al. 1971) which revealed a 17\% growth in membrane thickness at the liquid crystalline $\left(\mathrm{L}_{\alpha}\right)$ to the gel $\left(\mathrm{L}_{\beta}\right)$ phase transition, due to the gauche - all-trans configuration transition of the fatty acid residues of lipids.

Interestingly, discrete and discontinuous changes (jumps) are observed between thermodynamic phases upon temperature variations. In the ordered phases $\left(\mathrm{P}_{\beta}\right.$, and $\left.\mathrm{L}_{\beta}\right)$, molecules are packed together tightly and the acyl chains are highly ordered and maximally extended so, the bilayer thickness of the $\mathrm{P}_{\beta}$, and $\mathrm{L}_{\beta}$ are greater than that of the $\mathrm{L}_{\alpha}$ phase (Wilkins, Blaurock et al. 1971), and consequently, the capacitance decreases step by step between the different phases throughout the cooling process. 


\section{Current fluctuation patterns, self-similarity parameter $(\alpha)$ and histograms of the amplitude of the electrical current across DPPC-hBLM under the percolation voltage intensity $\left(\mathbf{V}_{\text {th }}\right)^{1}$}
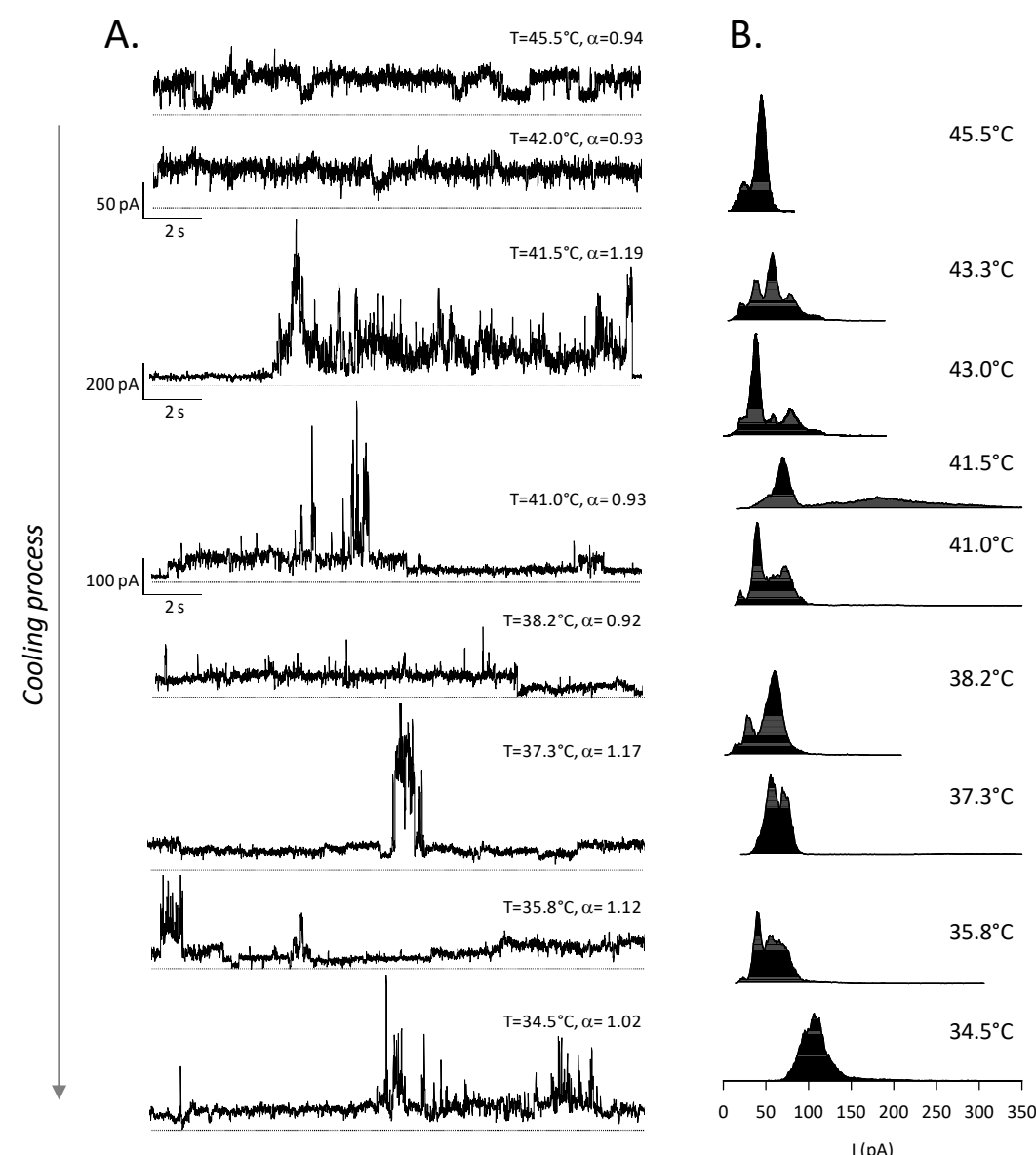

C.

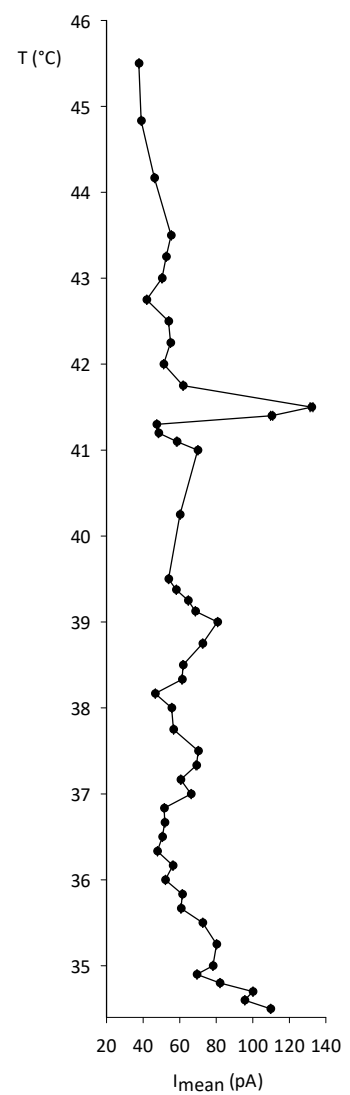

Fig.S4 Temporal-variation of I fluctuation pattern and self-similarity parameter $(\alpha)$ of DPPChBLM along the cooling process under the percolation voltage $\left(\mathbf{V}_{\text {th }}\right)$. A DPPC-hBLM, clampted to $160 \mathrm{mV}$ transmembrane voltage, was submitted to a continuous cooling from $45.5^{\circ} \mathrm{C}$ to $34.5^{\circ} \mathrm{C}$ within $980 \mathrm{~s}$ total time span, corresponding to a $0.67^{\circ} \mathrm{C} / \mathrm{min}\left(0.011^{\circ} \mathrm{C} / \mathrm{s}\right)$ cooling rate. Data acquisition was performed continuously. A) Representative transmembrane ionic current recordings from DPPC-hBLM upon application of a $160 \mathrm{mV}$ holding potential in the presence of symmetric $\mathrm{K}+/ \mathrm{Cl}-(150 \mathrm{mM} \mathrm{KCl})$. Numbers in the figure indicate the temperature and the corresponding $\alpha$ value calculated within the ionic current interval analyzed. Dotted lines represent the baseline (equivalent to $\mathrm{I}=0 \mathrm{~A}$ ). B) Amplitude histograms obtained by fitting a Gaussian distribution function to experimental I values at different temperatures (specified in the figure) and spanned along $20 \mathrm{~s}$ time ranges. In these conditions, it can be assumed that each plot is calculated at an almost constant temperature $\left( \pm 0.1^{\circ} \mathrm{C}\right)$. C) Average I values ( $\mathrm{I}_{\text {mean }}$ ) computed for consecutive $20 \mathrm{~s}$ intervals vs. Temperature $\left({ }^{\circ} \mathrm{C}\right)$ plot.

\footnotetext{
${ }^{1}$ Data show a wider $I$ distribution at temperatures close to the main transition temperature $\left(\mathrm{P}_{\beta}, \rightarrow \mathrm{L}_{\alpha}\right)(\mathrm{B})$ and a tendency towards higher $I$ values with decreasing temperature (C).
} 


\section{Detrended fluctuation analysis of temporal I patterns}

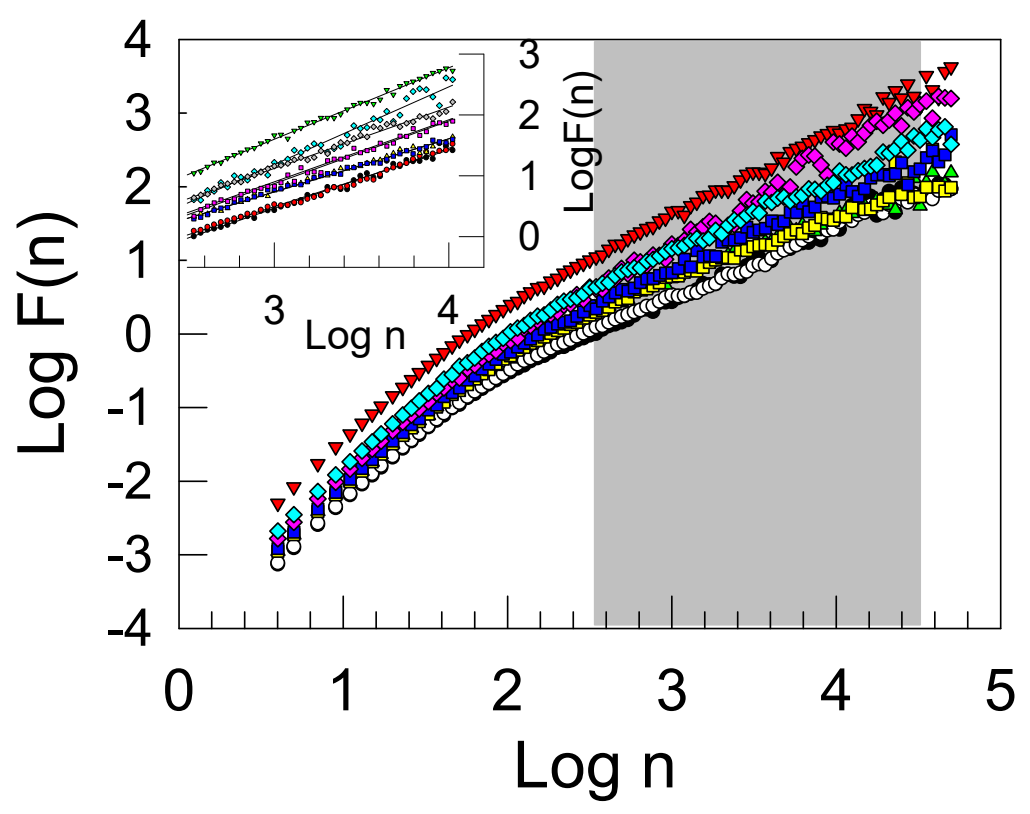

Fig.S5 Detrended fluctuation analysis of temporal I patterns scanned in DPPChBLM set at $\Delta V=160 \mathrm{mV}$. $\alpha$ was calculated from the slopes within the shaded region $(\log n=2.46-4.5)$.

\section{References}

Antonov, V. F., A. A. Anosov, V. P. Norik, E. A. Korepanova and E. Y. Smirnova (2003). "Electrical capacitance of lipid bilayer membranes of hydrogenated egg lecithin at the temperature phase transition." Eur Biophys J 32(1): 55-59.

Corvalán, N. A., J. M. Kembro, P. D. Clop and M. A. Perillo (2013). "Cholesterol favors the emergence of a long-range autocorrelated fluctuation pattern in voltage-induced ionic currents through lipid bilayers." Biochimica et Biophysica Acta (BBA) - Biomembranes 1828(8): 17541764.

Tarek, M. (2005). "Membrane Electroporation: A Molecular Dynamics Simulation." Biophysical journal 88(6): 4045-4053.

Tieleman, D. P. (2004). "The molecular basis of electroporation." BMC biochemistry 5(1): 10.

Toyama, S., A. Nakamura and F. Toda (1991). "Measurement of voltage dependence of capacitance of planar bilayer lipid membrane with a patch clamp amplifier." Biophys J 59(4): 939-944.

Wilkins, M., A. Blaurock and D. Engelman (1971). "Bilayer structure in membranes." Nature New Biology 230(11): 72-76. 